\title{
Accurate capture of propeller-rudder interaction using a coupled blade element momentum-RANS approach
}

\author{
Alexander B. Phillips*, Stephen R Turnock* and Maaten Furlong** \\ *Fluid-Structure Interaction Research Group, School of Engineering Sciences, University of Southampton, \\ Highfield, Southampton SO17 1BJ, UK \\ **National Oceanography Centre, Southampton, European Way, Southampton, SO14 3ZH, UK
}

Corresponding author's email: abp@soton.ac.uk

\begin{abstract}
The physically correct capture of propeller-rudder interaction is important if ship self-propulsion parameters are to be accurately predicted. Full resolution of a time accurate computational fluid dynamics calculation based on the Reynolds Averaged Navier Stokes equations requires a significant computational resource. The axial and tangential accelerations generated in the flow by the passage of the propeller blades may be represented by momentum source terms in the form of a body force propeller model. This paper compares experimental results with numerical simulations using three different body force propeller model approaches. The results demonstrate that as long as the radial variation in axial and tangential momentum generated by the propeller are included, then the influence of the unsteady propeller flow can be removed and 'steady' calculations performed to evaluate the influence of the propeller on the rudder.
\end{abstract}

\section{Introduction}

Axial and tangential accelerations applied to a fluid by the action of the propeller, lead to significant variations in the flow field around the stern of a vessel, compared to a towed body. Firstly, by accelerating the flow the local pressure is reduced increasing the pressure drag acting on the body. Secondly, since a ship rudder is typically placed downstream of the propeller, the rudder experiences accelerated axial and tangential inflow conditions compared to freestream. Several authors have proposed investigating the influence of the propeller on a hull or rudder by replicating the flow integrated effects of the propeller within a RANS simulations as a series of momentum source terms. Schetz and Favin [1] implemented an actuator disc propeller model within a RANS simulation calculating the flow around a $2 \mathrm{D}$ section of the stern of an axi-symmetric body. The propeller model allowed arbitrary variations in thrust distribution but swirl was assumed to be negligible. An increase in the $u$ velocity component upstream and downstream of the model was observed.

Stern et al. 2] considered the flow over the stern of an axis-symmetric body (Afterbody 1 ) with propeller (4577). The use of prescribed and interactive body force distributions were analysed using axial momentum terms with and without swirl momentum. The prescribed body forces used thrust and moment distributions based on the radial circulation distributions presented by Hough and Ordway 3. This distribution was shown to match Goldstein's optimum distribution.This method ignored the interaction of the propeller and hull. The interactive body force distribution was calculated for the propeller thrust and torque $\left(K_{T}\right.$ and $K_{Q}$ ) using a vortex based lifting surface method.

Simonsen 44 performed RANS simulations of the propeller-rudder experiments of Molland and Turnock [5] using the academic solver CFDSHIP-IOWA with the same Hough and Ordway body force propeller model. Neither the propeller support geometry, hub or floor boundary layer were considered in this analysis. The simulations were able to capture the correct behaviour with respect to increasing rudder aspect ratio or changing thrust loading. The predictions of global integrated quantities showed that both the lift and drag were marginally under-predicted. This method is only able to consider the effects of the propeller on the rudder. The influence of the rudder on the propeller is neglected.

These methods assume that, if the radial variation in axial and tangential momentum (including hull and rudder interaction effects) generated by the propeller are included, the influence of the unsteady propeller flow can be removed and 'steady' calculations performed to evaluate propeller hull or propeller rudder interaction. As the rudder blocks and diverts the flow through the propeller, an improved method calculates the axial and tangential momentum source terms based on the Blade Element Momentum Theory (BEMT) of 6]. This has been coupled to the commercial RANS solver CFX. It can be used to account for radial and circumferential variations in axial and tangential wake due to the presence of hull and rudder, [7, 8].

In this work the ability of three different body force propeller models to replicate propeller rudder interaction will be considered. The numerical results are compared with the wind tunnel experiments by Molland and Turnock [9, 10, 11, using their modified Wageningen B4.40 propeller and a rudder designated Rudder No.2. 
The flow around a rotating propeller is a complex transient flow. Time accurate capture of a rotating propeller explicitly leads to significant computational cost within a RANS simulation. This is due to the high mesh resolution required around the blade to capture the flow features and the small time steps required to capture the transient flow behaviour. For work not concentrating on the propeller itself but rather the interaction of the propeller hull and rudder system it may not be vital to capture all aspects of the propeller flow. However, providing a representative model of the velocity field is extremely important. Based on this assumption several body force propeller models have been proposed to reduce computational cost, which facilitate self propelled ship simulations.

When using a body force model the propeller is not physically represented by its geometry. Instead the integrated effect of the propeller on the flow is included by representing the propeller as a series of axial and tangential momentum source terms, $f b_{x}$ and $f b_{\theta}$ respectively, which are distributed over the propeller disc, these induce an axial and swirl acceleration in the fluid.

In order to implement a body force propeller model in a RANS simulation both the magnitude and distribution of the thrust, $T$, and torque, $Q$, induced by the propeller needs to be determined. Three different bodyforce models will be considered:-

1. Uniform thrust distribution with no torque, equivalent to an actuator disc applied over a finite thickness.

2. Hough and Ordway prescribed thrust and torque radial distribution.

3. Thrust and torque magnitude and radial and tangential distributions from BEMT.

Typical velocity profiles available in the literature, show that the circumferentially averaged velocity in the radial direction is an order of magnitude smaller than the circumferentially averaged velocity component. Kee et al. [12] found that the circumferentially averaged radial velocity was $15 \%$ of the circumferentially averaged axial velocity component for a similar propeller operating at bollard pull $(J=0)$. Hence, it is assumed that no tangential source terms are required and that no significant alteration is required to the axial and tangential momentum source terms. Radial velocity changes due to contraction of the slipstream are captured through the mass continuity condition directly by the solution of the RANS equations.

Equations (1) and (2) are the incompressible RANS equations in tensor form. When using the body force propeller models described the momentum source terms $f_{i}$ are set to zero except within a cylindrical region of diameter $D$ located at the propeller disc of a finite length typically $0.1 \mathrm{D}$.

$$
\frac{\partial \overline{U_{i}}}{\partial x_{i}}=0
$$

$$
\begin{aligned}
\rho \frac{\partial \overline{U_{i}}}{\partial t}+\rho \frac{\partial \overline{U_{i} U_{j}}}{\partial x_{j}}= & -\frac{\partial P}{\partial x_{i}}+\frac{\partial}{\partial x_{j}}\left\{\mu\left(\frac{\partial \overline{U_{i}}}{\partial x_{j}}+\frac{\partial \overline{U_{j}}}{\partial x_{i}}\right)\right\} \\
& -\rho \frac{\partial \overline{u_{i}^{\prime} u_{j}^{\prime}}}{\partial x_{j}}+f_{i}
\end{aligned}
$$

The influence of turbulence on the mean flow is represented in equation (2) by the Reynolds stress tensor $\left(\rho \overline{u_{i}^{\prime} u_{j}^{\prime}}\right)$

Closure of the RANS equations is achieved for this case using the Shear Stress Transport (SST) model eddy viscosity turbulence closure model developed by [13. SST is a two zone model that blends a variant of the $k-\omega$ model in the inner boundary layer with a transformed version of the $k-\varepsilon$ in the outer boundary layer and away from the wall. Previous investigations for ship flows have shown it is better able to replicate the flow around ship hull forms than either zero equation models or the $k-\varepsilon$ model, notably in capturing hooks in the wake contours at the propeller plane, [14, 15].

\subsection{Uniform Thrust Distribution - RANS-UT}

The simplest approach to replicating a propeller with a known thrust is to assume a uniform distribution of thrust over the propeller disc. The torque is neglected. The axial momentum source term is given by:-

$$
f b_{x}=\frac{T}{\Delta x \pi\left(R_{p}^{2}-R_{h}^{2}\right)},
$$

while the tangential momentum term is given by:-

$$
f b_{\theta}=0,
$$

where $\Delta x$ is the thickness of the propeller subdomain and $R_{h}$ and $R_{p}$ are the radius of the hub and propeller respectfully. The magnitude of $T$ must be provided by some other means, either experimental, based on open water data or from numerical predictions.

\subsection{Hough and Ordway Thrust and Torque Distribu- tion - RANS-HO}

The radial distribution of thrust and torque is based on the 3] circulation distribution which has zero loading at the tip and root. The distribution was shown to match Goldstein's optimum distribution. Coupling this distribution with a RANS simulation was proposed by [2], and is implemented in CFDSHIP-IOWA, [16. The non-dimensional thrust distribution $f b_{x}^{\prime}$ and torque distribution $f b_{\theta}^{\prime}$ are given by:-

$$
\begin{gathered}
f b_{x}^{\prime}=A_{x} r^{*} \sqrt{1-r^{*}} \\
f b_{\theta}^{\prime}=A_{\theta} \frac{r^{*} \sqrt{1-r^{*}}}{\left(1-Y_{h}\right) r^{*}+Y_{h}}
\end{gathered}
$$

where:

$$
A_{x}=\frac{C_{T}}{\Delta x} \frac{105}{16\left(4+3 Y_{h}\right)\left(1-Y_{h}\right)}
$$




$$
A_{\theta}=\frac{K_{Q}}{\Delta x J^{2}} \cdot \frac{105}{\pi\left(4+3 Y_{h}\right)\left(1-Y_{h}\right)}
$$

where the non dimensional radius is defined as $r^{*}=$ $\left(Y-Y_{h}\right) /\left(1-Y_{h}\right), Y=r / R_{p}$ and $Y_{h}=R_{h} / R_{p}$.

$$
C_{T}=\frac{T}{1 / 2 \rho U_{a}^{2} \pi R_{p}^{2}}=\frac{K_{T}}{\pi / 8 J^{2}}
$$

For uniform propeller inflow such as that experienced by a propeller rudder system operating in freestream, 4 demonstrated that the use of this thrust and torque distribution lead to good estimates of rudder forces.

2.3 Blade Element Momentum Theory, Thrust and Torque Magnitude and Distribution Prediction RANS-BEMT

The thrust and torque distribution will vary depending on the geometry of the propeller, rpm and advance velocity. To incorporate these variations the thrust and torque magnitude and distribution are calculated using an existing BEMT code [6].

In order to capture the radial and circumferential variation in propeller inflow conditions due to blockage effects from the rudder, the propeller plane is subdivided into for 360 discrete zones (10 radial divisions, 36 circumferential divisions). The BEMT code is called for each of these locations to determine the local $K_{T}$ and $K_{Q}$ based on the segmented inflow conditions from an initial converged simulation with the propeller model turned off. For each node in the propeller subdomain, the radial distance is calculated and the local thrust and torque magnitudes are interpolated from the distribution derived from the BEMT code. Further details of this approach may be found in [17, 7, 8].

\section{Experimental Data}

The wind tunnel tests performed by Molland and Turnock in the University of Southampton $3.5 \times 2.5 \mathrm{~m}$ RJ Mitchell Wind Tunnel [9, 18]. The experimental set up is shown in Figure 1. It comprises a $1 \mathrm{~m}$ span, 1.5 geometric aspect ratio rectangular planform square tipped rudder (No. 2) with a constant NACA0020 section, and a representative $0.8 \mathrm{~m}$ diameter propeller based on the Wageningen B4.40 series placed at $X / D=0.39$, where $X$ is the distance between the propeller plane of revolution and the leading edge of the rudder at zero degrees incidence. Further details of the experiment can be found in 11 .

\section{Numerical Model}

Simulations are performed using ANSYS CFX V11, [19], a commercial, fully implicit finite volume code, using a variation of the SIMPLE (Semi-Implicit Method for Pressure Linked Equations) algorithm for momentum-pressure decoupling. Details of the computational parameters are provided in Table 1 .

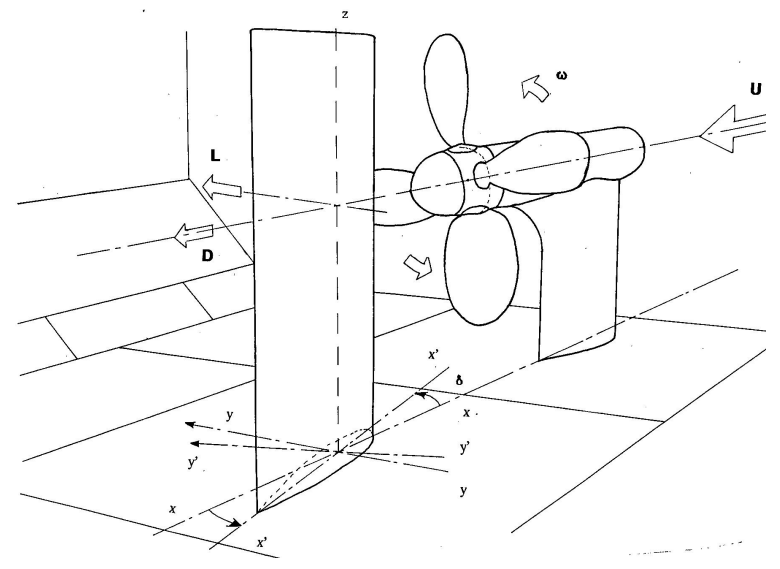

Figure 1: Isometric view of experimental setup, 5]

\begin{tabular}{|c|c|}
\hline Parameter & Setting \\
\hline Mesh Type & Unstructured with local \\
\hline & refinement in vortical regions \\
\hline No. of Elements & approximately $10 \mathrm{M}$ \\
\hline Computing & Iridis 2 Linux Cluster \\
\hline Run Type & $\begin{array}{l}\text { Parallel ( } 8 \text { partitions run on } \\
4 \times \text { dual core nodes } \\
\text { each with } 2 \text { Gb RAM) }\end{array}$ \\
\hline Turbulence Model & Shear Stress Transport \\
\hline & $30-60$ on rudder, 200 on floor \\
\hline Wall Modelling & Automatic Wall Functions \\
\hline Spatial & \\
\hline Discretisation & High Resolution [19] \\
\hline Pseudo Time Step & $0.1 \mathrm{~s}$ \\
\hline Convergence & \\
\hline Control & RMS residual $<10^{-5}$ \\
\hline Simulation Time & Typically $2.5-3 \mathrm{hrs}$ \\
\hline
\end{tabular}

Table 1: Computational Parameters

\subsection{Model Domain and Boundary Conditions}

The lateral dimensions were matched to those of the $11^{\prime} \times 8^{\prime}$ RJ Mitchell wind tunnel, with the domain extending 2.8 chord lengths upstream of the rudder and 8 chord lengths downstream. The following boundary conditions were applied:

- Inlet - Dirchlet boundary condition, nominal inflow velocity $10 \mathrm{~m} / \mathrm{s}$, with representative $70 \mathrm{~mm}$ thick boundary layer distribution applied over floor, turbulence intensity 0.002, eddy length scale $0.01 \mathrm{~m}$.

- Outlet - Neumann boundary condition, velocity and pressure gradients set to zero, with a zero relative pressure. The boundary condition selected will impact the flow upstream of the outlet, especially the low pressure regions in the vortex core which will be forced to zero relative pressure on the outlet. However, this influence will only act a short distance upstream and minimal impact should be experienced on the solution of rudder loads or surface pressure distributions due to the 
outlet boundary condition

- Wind tunnel floor - no slip, initial studies neglecting the floor boundary layer were unable to replicate the flow at the root of the rudder.

- Wind tunnel walls - free slip, wall boundary condition.

- Rudder - no slip, wall boundary condition.

- Hub - no slip, wall boundary condition.

Within the RANS simulation the propeller is modelled as a cylindrical subdomain with a diameter equal to that of the propeller and a length equal to that of the rotating hub. Momentum source terms are then applied over the subdomain in cylindrical co-ordinates to represent the axial and tangential momentum induced by the propeller, as calculated by the three body force propeller models.

\section{Flow Feature Identification and Mesh Refinement}

The structure of the flow downstream of a rudder operating in freestream conditions is well understood. However, the influence of the propeller upstream results in a highly complex flow, the tip vortex generated by the rudder acts to break up the propeller race. Since the race itself incorporates a large swirl component as the wake is broken up it has a tendency to roll up into a series of secondary vortices. The number and path of which cannot be predicted a priori.

To track these vortex structures the VORTFIND algorithm 20] is used. The VORTFIND algorithm is a robust and computationally inexpensive method of identifying the vortex core centre using just the velocity data on a transverse plane, and is extended to three dimensions through using a series of planes that are normal to the local vortex direction, 21. It is a line method, that is not Galilean invariant but allows multiple vortices to be captured, only requiring knowledge of the velocity field. The original VORTFIND algorithm has been successfully used to identify bilge vortices, control surface tip vortices and propeller tip vortices. Modifications to the algorithm to make it more robust when identifying multiple vortex structures are detailed in 22 .

In order to ensure suitable spatial resolution of the vortex structures an iterative unstructured mesh strategy is used.

1. An initial coarse mesh is produced with approximately 100000 elements in the propeller domain and 1.5 million elements in the far field. A separate mesh is built for each rudder angle, which is used for the three different propeller model approaches. The resulting flow field contains diffuse flow features but enables regions of interest to be identified. At a series of tangential cut planes downstream of the the rudder the lateral and vertical extents of the propeller race are identified. The extent of the propeller race is determined as the location where $u / U_{0}>1.0$.
2. Script files then take the extents of the wake and enter this data into the commercial meshing code ANSYS ICEM CFD V11 as a series of regions with increased mesh density. A second finer mesh is built with an approximate far field mesh size of 4.0M elements. The lateral extent of the propeller race is different for each of the three propeller models. Thus, a different mesh must be built for each case. The preliminary coarse mesh has insufficient elements to capture the flow features downstream of the rudder, to a suitable level of precision. The lack of elements leads to numerical diffusion of the features resulting in weaker larger structures than a mesh resolved solution. By placing more elements in the region of the propeller race, a better estimate of the extent of these flow features can now be achieved.

3. The results from these secondary meshes are examined more closely, the rudder tip vortex and secondary vortex structures are identified using the modified Vortfind algorithm.

4. Finally a final fine mesh is built placing finer mesh in the regions of the propeller race, tip vortex ( the tip vortex is assumed to originate at $2 / 3 \mathrm{rd}$ chord at the junction between the tip and pressure surfaces), secondary vortices and rudder boundary layer.

Figure 2 illustrates the downstream mesh refinement process. The initial coarse mesh results in highly diffused flow structures. The tip vortex is evident in the lateral vector plot but none of the secondary vortices have propagated this far downstream $X / D=7.0$ $(X / D=0$ at the propeller plane). However the initial mesh provides a good first estimate of the extents of the propeller race.

\section{Results and Discussion}

\subsection{Momentum Source Terms}

Figures 3 and 4 illustrate the applied momentum source terms in the axial and radial direction respectively over the propeller domain for the three body force approaches for $J=0.34$ and $J=0.51$, without the presence of the rudder. The uniform thrust model applies uniform axial momentum source terms across the propeller diameter from hub to root. The magnitude of the source term reduces from $1890 \mathrm{Nm}^{-3}$ to $725 \mathrm{Nm}^{-3}$ for $J=0.34$ (high thrust loading) to $J=0.51$ (typical design thrust loading), this model neglects the induced tangential velocities and hence the tangential source term are zero. Both the Hough and Ordway and BEMT method, result in radially varying axial and tangential momentum source terms, varying from zero at the root and tip. The Hough and Ordway model induces a peak at $0.72 R$, while the BEMT method has a peak at $0.58 R$. It is important to highlight that the thrust distribution along the blade calculated by the BEMT code has a peak at $0.75 R$. 
However, once this is weighted by the propeller disc area, the peak is moved nearer to the hub.

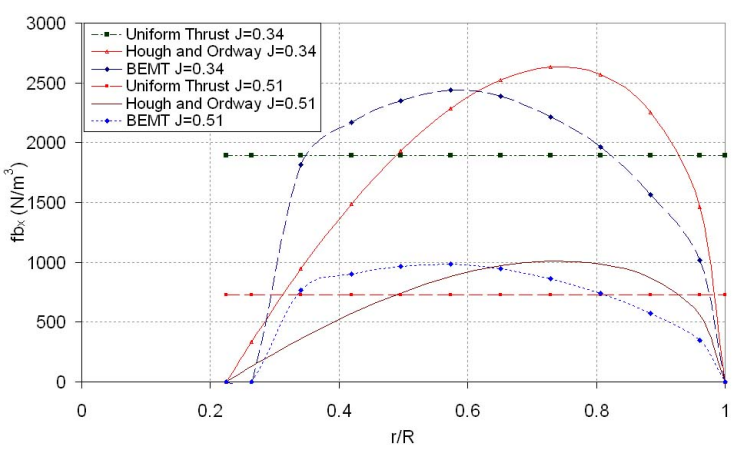

Figure 3: Radial distribution of axial momentum source terms for $\mathrm{J}=0.34$ and $\mathrm{J}=0.51$.

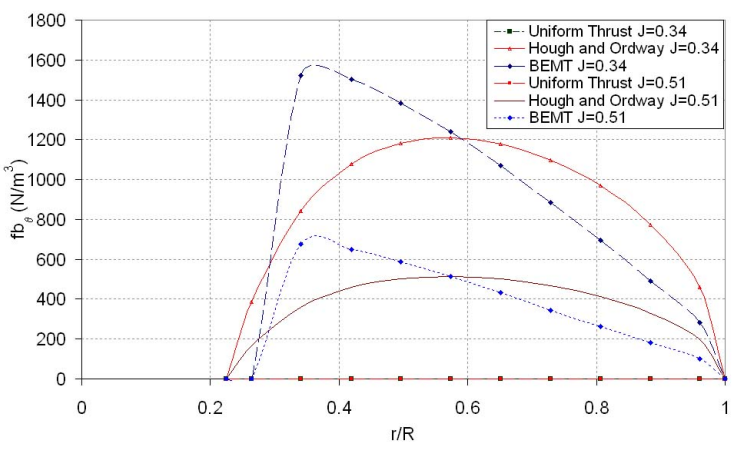

Figure 4: Radial distribution of angular momentum source terms for $\mathrm{J}=0.34$ and $\mathrm{J}=0.51$.

\subsection{Lift and Drag Data}

Results are presented below for the modified B4.40 propeller operating at the heavily loaded condition of $J=0.35$. $K_{T}$ and $K_{Q}$ for the Uniform Thrust and Hough and Ordway models was taken from open water data for the modified B4.40 propeller [23], to account for the blockage effects due to the rudder a $\delta K_{T}$ term was also applied.

Figure 5 and 6 compares the experimental and numerical lift and drag respectively. Results are also presented from [4] who performed similar numerical simulations using the CFDSHIP-IOWA code using a Hough and Ordway thrust and torque distribution, and from [24] using the 3D panel code Palisupan, 25. Table 2 compares values of $d C_{L} / d \delta$ and the zero lift incidence angle $\delta_{0}$.

By neglecting the influence of propeller induced swirl on the fluid, the RANS-UT model produces symmetric results at $\pm 10^{\circ}$ and the rudder neutral angle is at $0^{\circ}$. However the magnitude of the lift results provide good indicative results, the gradient of the lift slope is within $10 \%$ of mean experimental value. For this case the drag experienced by the rudder is significantly greater than the experimental results for the
RANS-UT model. This is due to neglecting the influence of swirl on local incidence angle. Experimental results show a decrease in effective rudder drag with increasing propeller thrust loading.

The RANS-HO and RANS-BEMT approaches show very good correlation with the experiential lift results in terms of both neutral rudder angle, $\delta_{0}$, and lift slope gradient. The asymmetry due to the swirl action of the propeller is well reproduced.

Table 2: Rudder lift performance.

\begin{tabular}{|c|c|c|}
\hline Data Set & $\frac{d C L}{d \delta}$ & $\delta_{0}$ \\
\hline Molland and Turnock SS46 & 0.132 & 0.093 \\
Molland and Turnock SS90 & 0.136 & 0.526 \\
Turnock (1993) & 0.140 & 1.376 \\
Simonsen (2000) & 0.147 & 1.383 \\
RANS-UT & 0.123 & 0.000 \\
RANS-HO & 0.136 & 0.213 \\
RANS-BEMT & 0.139 & 0.227 \\
\hline
\end{tabular}

\subsection{Rudder Surface Pressure}

The ability of the three propeller models to replicate the downstream wake of a propeller can be inferred by their ability to replicate the correct pressure distribution on the rudder surface.

Examining the $C p=\frac{P-P_{0}}{1 / 2 \rho U_{0}^{2}}$ distribution for a freestream rudder, the maximum $C p$ has a value of 1.0 at the stagnation point. For a rudder downstream of a propeller the inflow velocity is greater than the freestream velocity, hence the stagnation pressure is higher leading to $C_{P}$ values in excess of 1 . Accurate prediction of stagnation $C_{P}$ values implies the correct inflow velocity has been generated by the propeller model.

Figure 7 compares experimental and numerical pressure distributions at a series of span locations from root to tip for a rudder angle of $\delta=0$.

The RANS-UT model experiences a symmetric pressure distribution down both sides of the rudder in stark contrast to the more complex models and the experimental data. Both the RANS-BEMT and RANS$\mathrm{HO}$ models reproduce relatively well the pressure distribution for most of the locations considered showing clear improvement over the RANS-UT approach. All three models fail to replicate the pressure distribution at $70 \mathrm{~mm}$ span. This location approximately corresponds with the top of the wind tunnel floor boundary layer. The failure to recreate the pressure distribution in this region implies that the interaction of the wind tunnel boundary layer with the rudder root and subsequent horseshoe vortex is poorly captured, for example see [26] for a discussion of required mesh resoloution.

Both the RANS-BEMT and RANS-HO approaches are relatively well able to replicate the pressure distribution at incidence angles of both 10 and -10 degrees, see Figures 8 and 9 respectively. Since the swirl imparted swirl direction is constant in both cases the 
resulting pressure fields are substantially different and this is reproduced in the numerical results.

\section{Conclusions}

The methods applied in this paper make use of the flow integrated effects of the propeller which generates an accelerated and swirled onset flow onto the rudder. As long as the radial variation in axial and tangential momentum generated by the propeller are included, then the influence of the unsteady propeller flow can be removed and 'steady' calculations performed to evaluate the influence of the propeller on the rudder.

The RANS-UT approach which neglects swirl results in adequate prediction of total rudder lift but is unable to predict the rudder drag. The use of this method is not recommended.

Both the RANS-BEMT and RANS-HO methods provided good estimates of the lift and the estimate of drag lies within the experimental uncertainty. Both, are also well able to replicate the pressure distribution acting on the rudder at $-10^{\circ}, 0^{\circ}$ and $10^{\circ}$ degrees. It is considered that prediction of the liftcurve slope $\left(\frac{d C_{L}}{d \delta}\right)$ within $5 \%$ of the experimental value is acceptable for parametric design investigations of propeller-rudder interaction. Consequently both the RANS-BEMT and RANS-HO model are considered as suitable methodologies for considering propellerrudder interaction with ship type propellers.

The RANS-HO approach is only suitable when investigating the influence of the propeller on the rudder. Interaction effects due to the rudder on the propeller are not captured, and neither would the influence of the hull on the propeller inflow conditions. Also the prescribed thrust and torque distributions are based on an infinitely bladed propeller. Consequently, the radial distribution of momentum source terms is not appropriate for low blade area ratio propellers.

The blockage effects of the rudder or influence of non uniform inflow into the propeller can be achieved using the coupled RANS-BEMT approach described, making it more suitable for cases involving hull-propellerrudder interaction such as self propulsion simulations.

\section{Acknowledgements}

Mr Phillips' PhD studentship is jointly financed by the School of Engineering Science and the National Oceanography Centre, Southampton.

\section{References}

[1] Schetz JA, Favin S. Numerical Solution for the Near Wake of a Body with a Propeller. Journal of Hydronautics 1977;11:136141.

[2] Stern F, Kim HT, Patel NM, Chen HC. A Viscous-Flow Approach to the Computation of Propeller-Hull Interaction. Journal of Ship Research 1988;32:246-262.

[3] Hough GR, Ordway DE. The generalised actuator disc. Developments in Theoretical and Applied Mechanics 1965;2:317336 .

[4] Simonsen CD. Rudder, Propeller and Hull Interaction by RANS. Ph.D. thesis. Department of Naval Architecture and Offshore Engineering; 2000.
[5] Molland AF, Turnock SR. Wind Tunnel Investigation of The Influence of Propeller Loading on Ship Rudder Performance. Ship Science Report No. 46; 1991.

[6] Molland AF, Turnock SR. A Compact Computational Method For Predicting Forces On A Rudder In A Propeller Slipstream. In: Transactions of RINA. vol. 138; 1996. p. 59-71.

[7] Phillips AB, Furlong M, Turnock SR. Comparisons of CFD Simulations and In-service Data for the Self Propelled Performance of an Autonomous Underwater Vehicle. In: 27th Symposium on Naval Hydrodynamics Seoul, Korea; 5-10 October 2008.

[8] Phillips AB, Turnock SR, Furlong M. Simulation of a selfpropelled ship using a Blade Element Momentum propeller model and RANS. Ocean Engineering 2009;36:1217-1225.

[9] Molland AF, Turnock SR. Influence of propeller loading on ship rudder performance. Transactions of the Royal Institution of Naval Architects 1992;135:105-120.

[10] Molland AF, Turnock SR. Wind Tunnel Tests on the Effect of a Ship Hull on Rudder-Propeller Performance at Different Drift Angles. University of Southampton Ship Science Report No. 76, May; 1995.

[11] Molland AF, Turnock SR. Marine Rudders and Control Surfaces. Butterworth-Heinemann; 2007.

[12] Kee C, Hamill GA, Lam WH, Wilson PW. Investigation of the Velocity Distributions within a Ships Propeller Wash. In: Proceedings of the Sixteenth (2006) International Offshore and Polar Engineering Conference San Francisco, California, USA, May 28-June 2,; 2006.

[13] Menter FR. Two-equation eddy-viscosity turbulence models for engineering applications. AIAA Journal 1994;32(8):1598 605 .

[14] Larsson L, Stern F, Bertram V. Benchmarking of Computational Fluid Dynamics for Ship Flows: The Gothenburg 2000 Workshop. Journal of Ship Research 2003;47:63-81(19).

[15] Hino T. CFD Workshop Tokyo 2005. In: The Proceedings of CFD Workshop Tokyo; 2005.

[16] Paterson EG, Wlison RV, Stern F. General Purpose parallel unsteady RANS ship hydrodynamics Code CFDSHIP-IOWA. Iowa Institute of Hydraulic Research, The University of Iowa; 2003.

[17] Turnock SR, Phillips AB, Furlong M. URANS Simulations Of Static Drift And Dynamic Manoeuvres Of The KVLCC2 Tanker. In: SIMMAN International Manoeuvring Workshop, Copenhagen; April 2008. .

[18] Molland AF, Turnock SR, Smithwick JET. Wind tunnel tests on the influence of propeller loading and the effect of a ship hull on skeg-rudder performance. University of Southampton, Ship Science Report 90; 1995.

[19] ANSYS. ANSYS CFX, Release 11.0. ANSYS; 2006.

[20] Pemberton RJ, Turnock SR, Dodd TJ, Rogers E. A Novel Method For Identifying Vortical Structures. Journal of Fluids and Structures 2002;16:1051-1057.

[21] Pashias C. Propeller Tip Vortex Simulation Using Adaptive Grid Refinement Based On Flow Feature Identification. Ph.D. thesis. University of Southampton; 2005.

[22] Phillips AB. Cost effective hydrodynamic design of Autonomous Underwater Vehicles. Ph.D. thesis. School of Engineering Sciences, University of Southampton; to be submitted 2009.

[23] Molland AF, Turnock SR. Wind tunnel test results for a model ship propeller based on a modified Wageningen B4.40. University of Southampton, Ship Science Reports 43; 1990.

[24] Turnock SR. Prediction of ship rudder-propeller interaction using parallel computations and wind tunnel measurements. Ph.D. thesis. University of Southampton, Ship Science; 1993.

[25] Turnock SR. A transputer based parallel algorithm for surface panel analysis. Ship Technology Research 1994;41:93-104.

[26] Pattenden RJ, Turnock SR, Zhang X. Measurements of the flow over a low-aspect-ratio cylinder mounted on a ground plane. Experiments in Fluids 2005;39:10-21. 


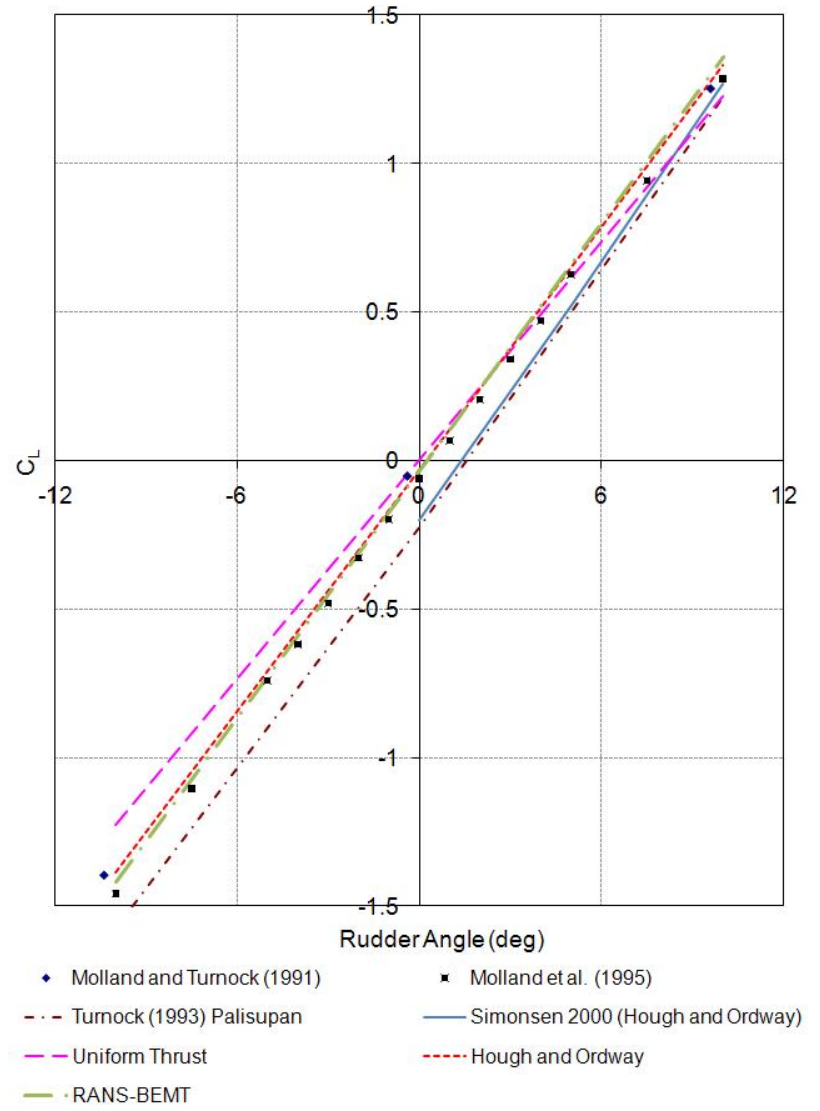

Figure 5: Rudder 2 - $C_{L}$ performance $J=0.35$

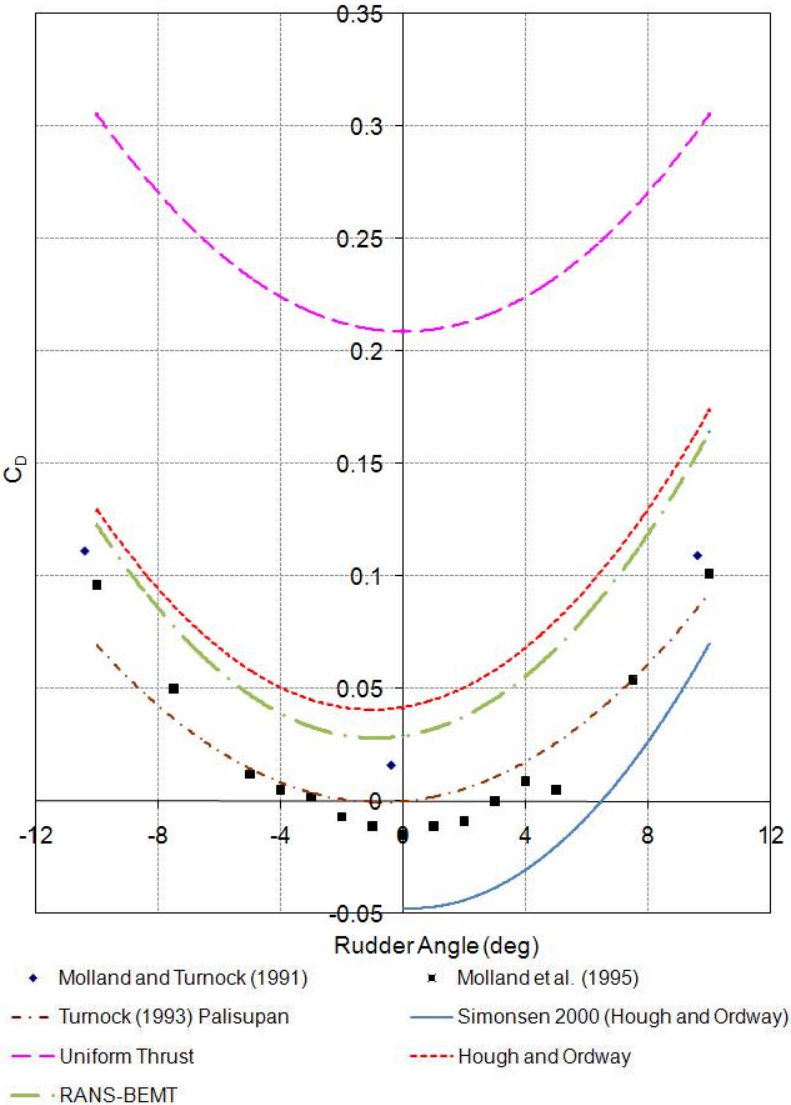

Figure 6: Rudder $2-C_{D}$ performance $J=0.35$ 


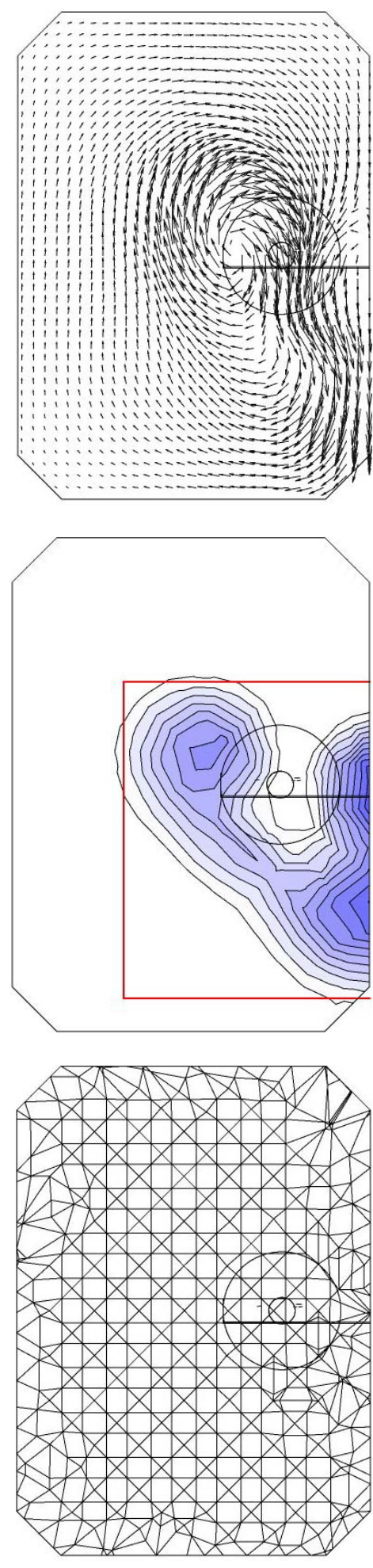

บSəNล [ย!ฺ!ฺI
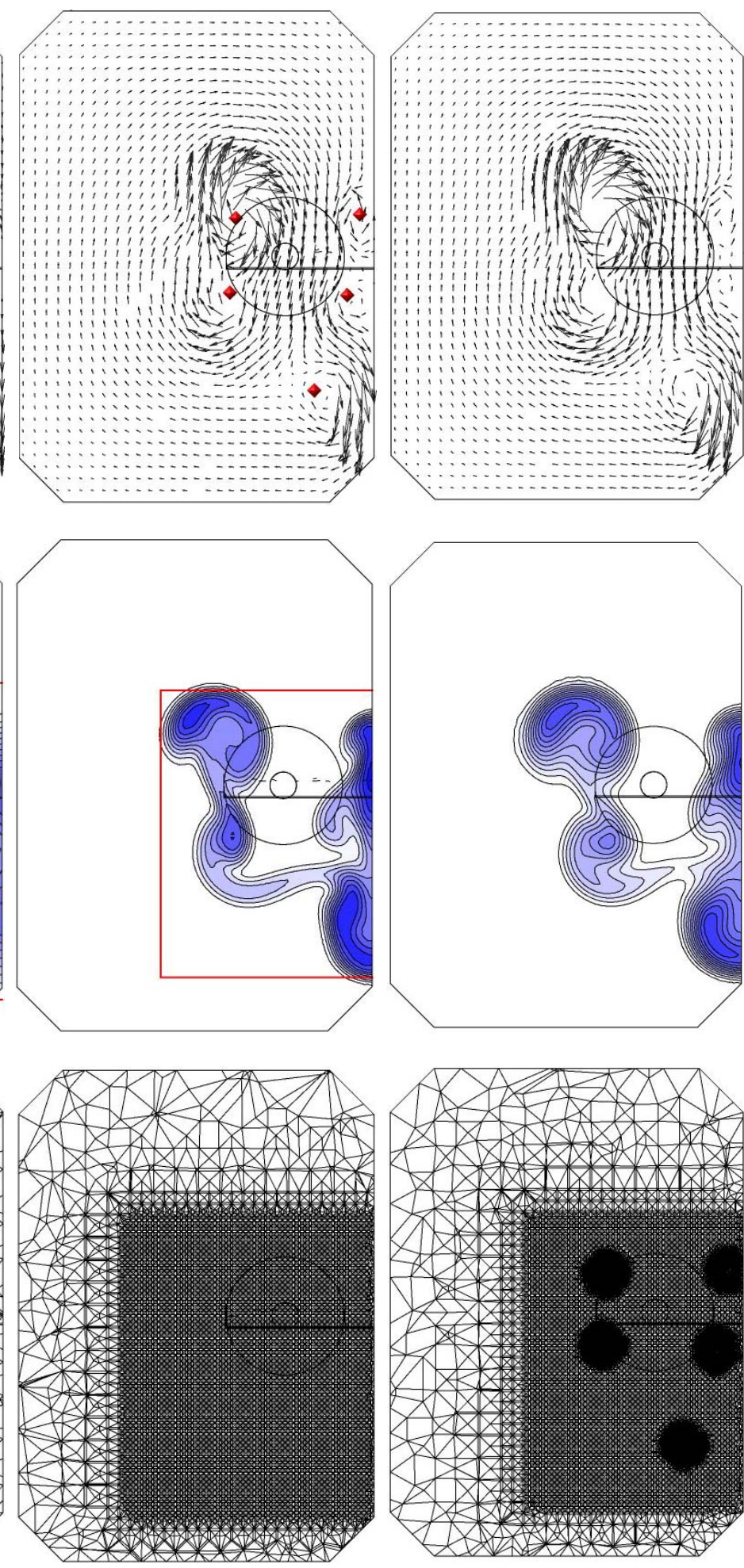

usəJN puz,

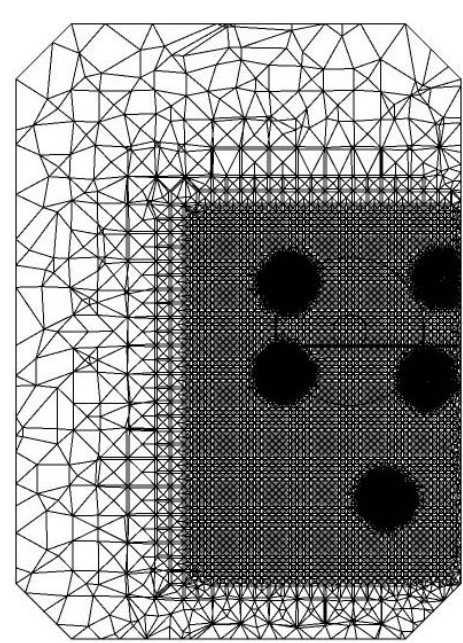

บsəN IอU!H 

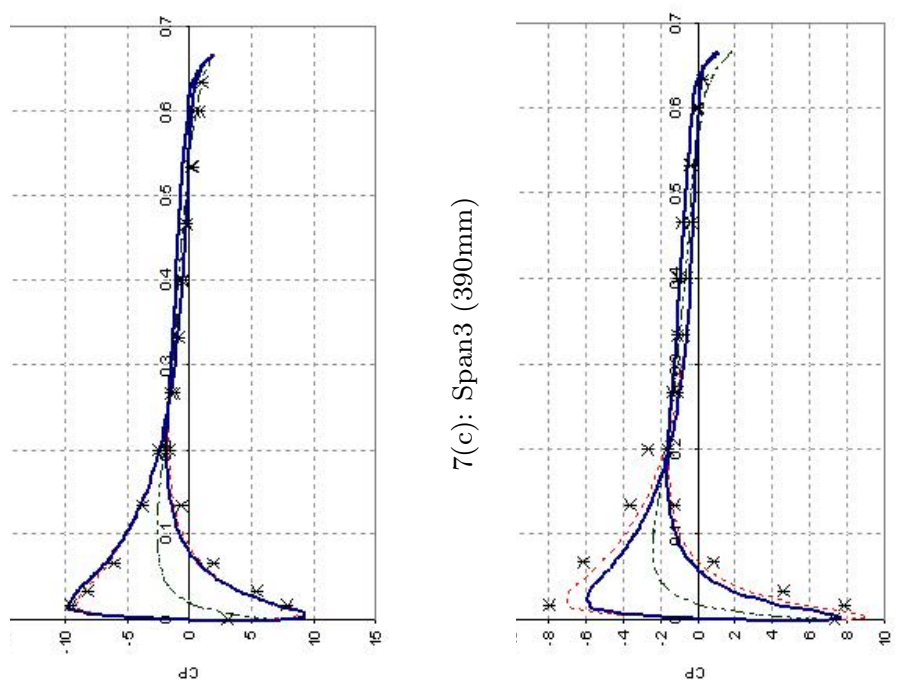

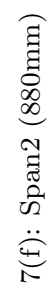
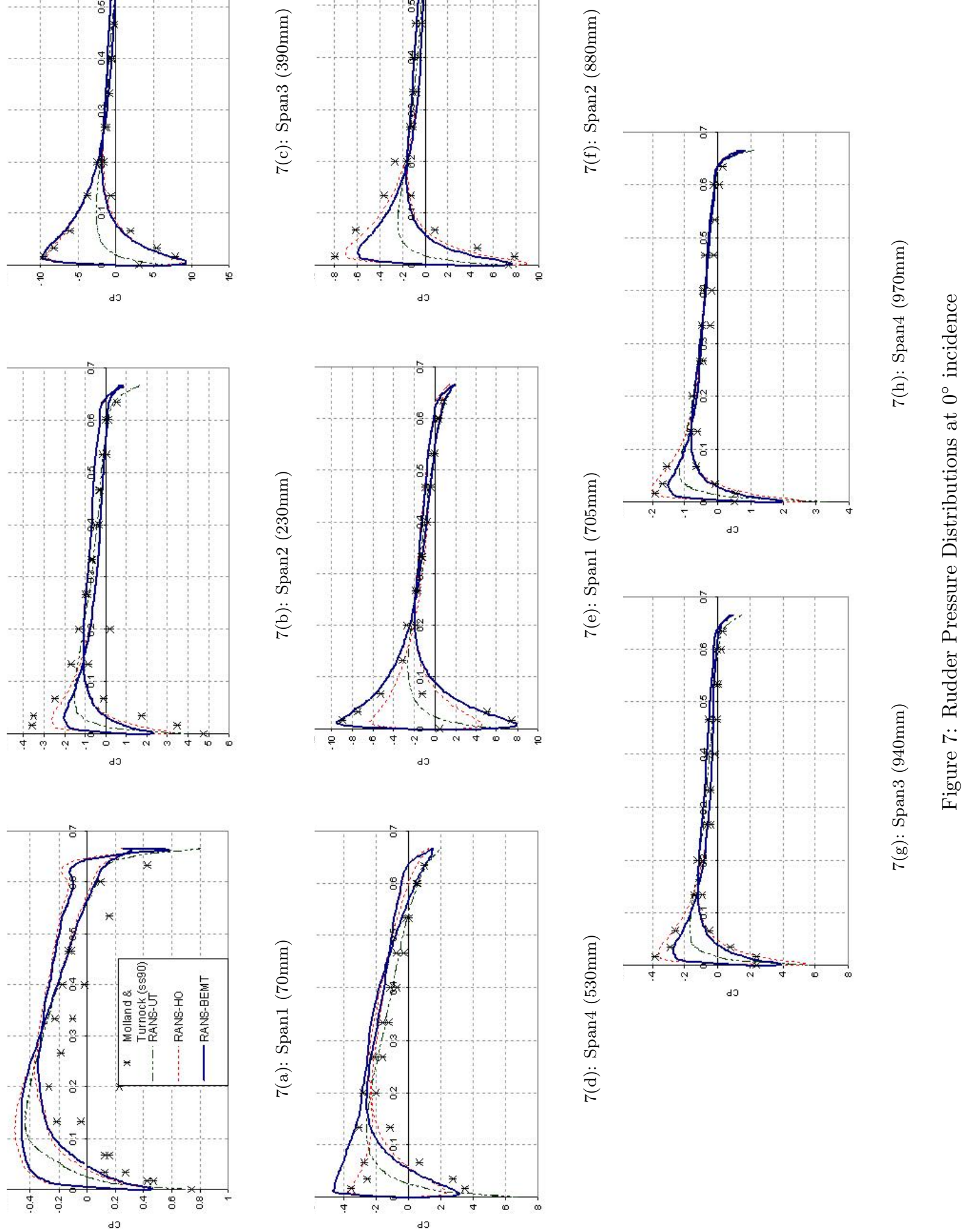

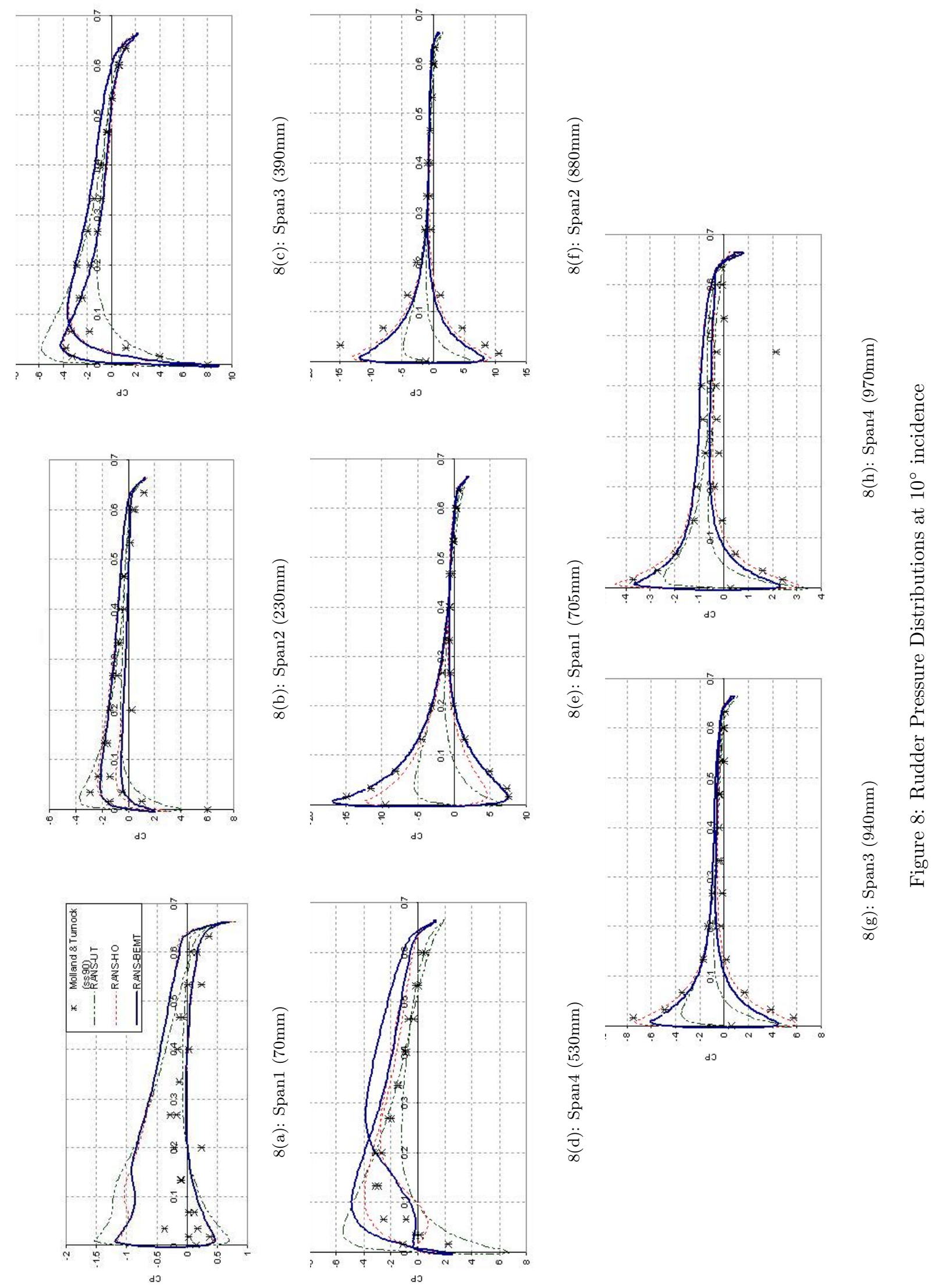

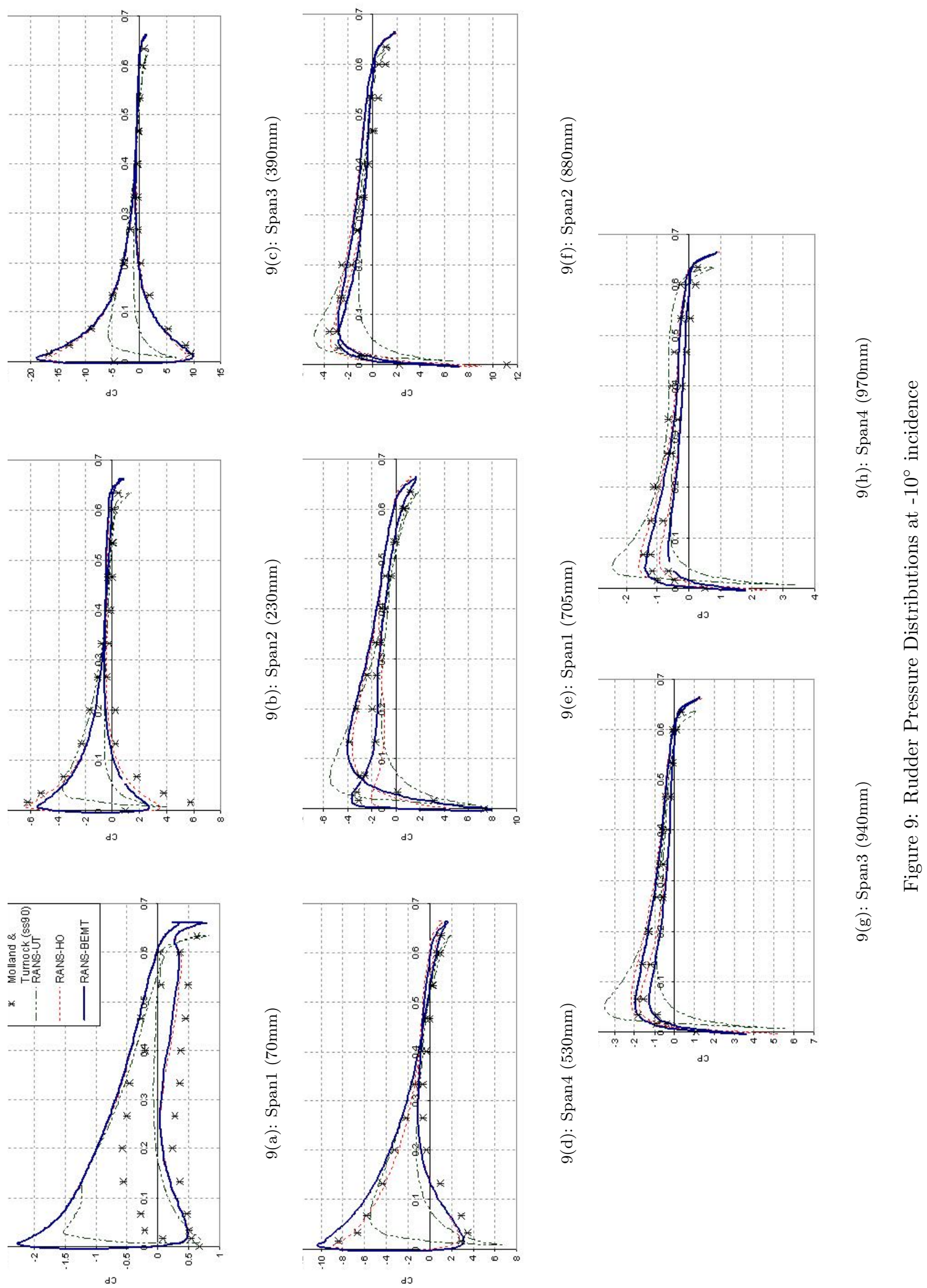